\title{
ESTATUS DE PESO Y CONDICIÓN FÍSICA: REVISIÓN DE LA LITERATURA CIENTÍFICA
}

\section{WEIGHT STATUS AND PHYSICAL FITNESS: REVIEW OF THE SCIENTIFIC LITERATURE}

\author{
Rosa-Guillamón, Andrés. ${ }^{1}$ \\ ${ }^{1}$ Rosa-Guillamón, Andrés. Doctor por la Facultad de Educación de la Universidad de Murcia \\ (Murcia, España) y Maestro de Educación Física en el CEIP Miguel Medina de Archena (Murcia, \\ España).andres.rosa@um.es
}

Código UNESCO: 2402.04 Composición del cuerpo

Clasificación Consejo de Europa: 17. Otras: Actividad Física y Salud

Recibido el 16 de octubre de 2017

Aceptado el 28 de noviembre de 2017

Correspondencia:

Gálvez Rosa-Guillamón, Andrés

andres.rosa@um.es

\section{RESUMEN}

La relación entre obesidad y condición física es un fenómeno recientemente estudiado y cuyo origen es motivo de discusión. Esta revisión sistemática trata de realizar una síntesis de los artículos científicos publicados en los últimos años que analizan la relación entre estatus de peso y condición física. La búsqueda incluye artículos publicados entre 2.006 y 2.017 , empleando para ello bases de datos electrónicas en inglés y español. Usando palabras clave como condición física, obesidad, estatus de peso, niños y adolescentes fueron incluidos artículos originales, artículos escritos en castellano o inglés, y estudios que incluían instrumentos de medida validados con anterioridad. En la primera búsqueda fueron hallados 159 artículos de los cuales un total de 16 artículos fueron seleccionados para la revisión. Los resultados obtenidos en estos artículos indican que la obesidad se asocia con una peor salud relacionada con la condición física.

PALABRAS CLAVE: actividad física, condición física, estatus de peso, niños, adolescentes. 


\begin{abstract}
The relationship between obesity and physical fitness is being recently studied and whose origin is still the reason of extensive controversy among those who research it. This review aims to summarize scientific studies published in the recent years that evaluate the relationship between weight status and physical fitness. The research includes studies that were published between 2.006 y 2.017 using English or Spanish electronic databases. Using keywords like physical fitness, obesity, weight status, children and adolescents. Included those items were written in Spanish or English, studies that included previously validated measurement instruments and original articles. In our first search a totally of 159 articles were found and finally a total of 16 articles were selected for inclusion in the review. The results indicate that obesity is associated with a poor health related to physical fitness.
\end{abstract}

KEYWORDS: physical activity, physical fitness, weight status, children, adolescents.

\title{
INTRODUCCIÓN
}

La condición física (CF) ha sido estudiada durante las últimas décadas por ser un excelente predictor de morbi-mortalidad por enfermedad cardiovascular y otras causas, independientemente del estado de salud de la persona, con o sin sobrepeso, tanto en varones ${ }^{1}$ como en mujeres ${ }^{2}$, siendo además considerado un bio-marcador de longevidad, calidad de vida y salud mental ${ }^{3-8}$. El descenso en los niveles de CF en la infancia y adolescencia es un hecho ${ }^{9}$. La evidencia científica disponible sugiere una relación directa entre un nivel bajo de CF y un estatus de obesidad en sujetos jóvenes ${ }^{10-13}$. Algunos estudios han puesto de manifiesto que la obesidad se encuentra directamente relacionada con diversos desórdenes fisiológicos ${ }^{14}$ e incluso psicosociales ${ }^{15-16}$. Esto tiene repercusiones muy negativas sobre la salud pública, por lo que es necesario diseñar intervenciones que hagan frente a esta epidemia global ${ }^{17-18}$. Para ello, es fundamental estudiar este fenómeno desde edades tempranas, ya que la infancia y la adolescencia constituyen un periodo fundamental en la adopción de las conductas que configuran estilo de vida durante la edad adulta. Algunas de estas conductas han sido relacionadas con la obesidad. Así sucede en concreto con los patrones de actividad física habitual ${ }^{19}$. Fomentar hábitos de estilo de vida saludable y evaluar el estatus de peso para detectar sujetos con bajos niveles de CF y obesidad puede ser primordial para evitar problemas de salud a medio o largo plazo. De ese modo, el objetivo de este trabajo fue clarificar la relación entre estatus de peso y condición física en escolares y adolescentes, así como sintetizar la literatura científica existente sobre la relación de dichas variables. 


\section{MATERIAL Y MÉTODO}

Se realizó una revisión sistemática de la literatura científica del fenómeno de estudio.

\section{Estrategia de búsqueda}

La búsqueda se basó en artículos publicados entre 2.006 y 2.015. La búsqueda bibliográfica se realizó en las siguientes bases de datos: Dialnet, Ebsco, Karger, Latindex, Pubmed, Medline, Science direct y Scielo. Las palabras clave empleadas fueron: actividad física, ejercicio físico, condición física, composición corporal, adiposidad, capacidad aeróbica, fuerza muscular, fitness, fitness cardiovascular, fitness muscular, consumo máximo de oxígeno $\left(\mathrm{VO}_{2}\right.$ máx.) rendimiento físico, obesidad, sobrepeso, normo-peso, índice de masa corporal, estatus de peso, estado nutricional, escolares, niños y adolescentes. También se utilizó la traducción de estas palabras al inglés.

La búsqueda fue realizada por primer autor. Se efectuó un seguimiento de la bibliografía de revisiones sistemáticas anteriores, con similares poblaciones objeto de estudio, para no perder información relevante no localizada en las bases de datos mediante el procedimiento descrito. Los artículos fueron examinados para determinar su inclusión o exclusión en el estudio.

\section{Criterios de inclusión}

La inclusión en la revisión se basó en los siguientes criterios: 1) Estar publicados entre 2.006 y 2.016; 2) Incluir poblaciones entre 6-19 años; 3) Analizar la relación entre composición corporal y condición física; 4) Ser artículos originales (estudios transversales, experimentales y cuasi-experimentales); 5 ) Estar escritos en castellano o inglés; 6) Incluir instrumentos de medida de contrastada fiabilidad y validez. Siguiendo los criterios mencionados, fueron incluidos un total de 16 artículos científicos (Figura 1). 
Figura 1. Diagrama de flujo de selección del proceso de selección.

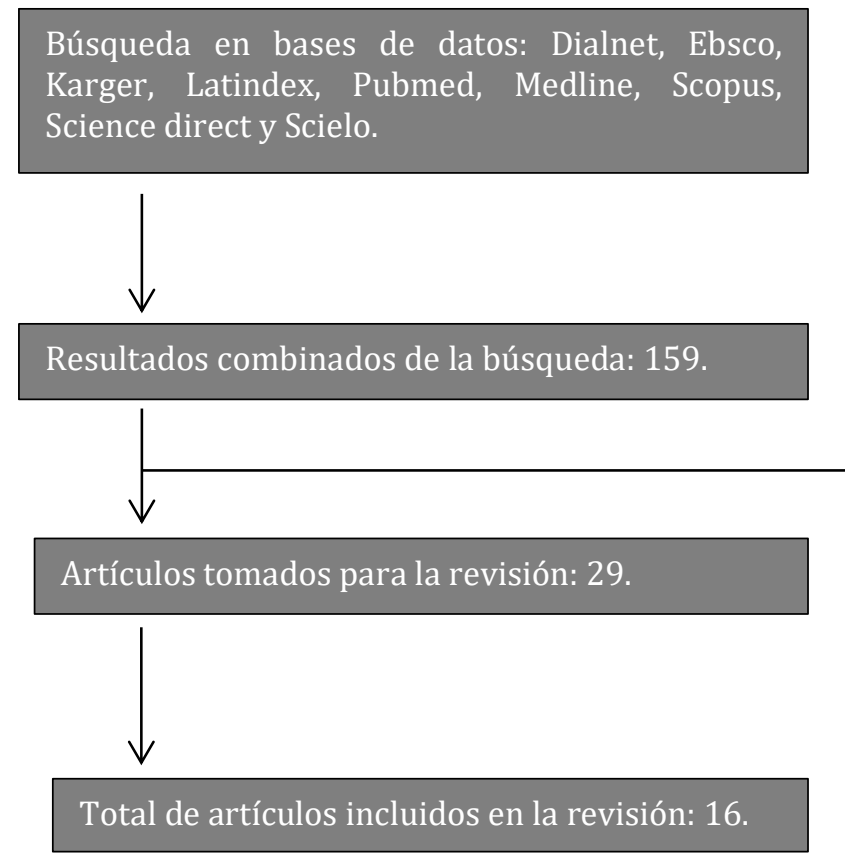

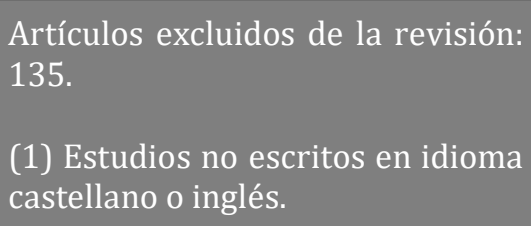

(2) Estudios que no incluían instrumentos de medida de contrastada fiabilidad y validez.

(3) Estudios que no incluyan el estatus de peso y la condición física.

(4) Estudios de revisión, metaanálisis y trabajos no publicados.

Fuente: elaboración propia.

\section{Gestión y extracción de datos}

El contenido de los artículos incluidos en el estudio fue extraído y resumido en los siguientes apartados: autor y año de publicación; tamaño de la muestra, edad y país; componentes/variables de la condición física; resultados más relevantes y conclusiones.

\section{RESULTADOS}

En la tabla 1 se presenta un resumen de los estudios incluidos en esta revisión (tabla 1).

En el estudio de Chen et al. ${ }^{20}$ se examinó la prevalencia de obesidad de una muestra de sujetos de Taiwán y se investigó la asociación entre el exceso de peso, la condición física y la presión arterial. Participaron en 1.999 a 7.031 varones y 6.904 mujeres, y en 2.001 a 12.367 varones y 12.219 mujeres de 618 años. Se categorizó a los sujetos según el estatus de peso: normo-peso, sobrepeso y obesidad ${ }^{21}$. Se midieron el peso y la talla. La condición física se midió con los test de encogimiento abdominal, sit and reach y subir y bajar a un cajón durante tres minutos. La presión arterial se midió con un esfigmomanómetro. Los resultados mostraron que el grupo con peso normal tuvo un mejor rendimiento físico en los test de encogimiento abdominal y en el test de subir y bajar el cajón. No hubo diferencias significativas en el test sit and reach. Durante el periodo de estudio, el riesgo de hipertensión aumentó casi dos veces para el grupo con exceso de peso pero en forma y casi tres veces para el grupo 
con exceso de peso pero no en forma, en comparación ambos con el grupo con normo-peso.

El trabajo de Casajús et al. ${ }^{22}$ estudió las diferencias en el rendimiento físico y la práctica física extracurricular de 1.068 sujetos categorizados con normopeso, sobrepeso y obesidad categorizado según su IMC ${ }^{21}$. La condición física fue medida con la batería Eurofit. Los sujetos con normo-peso tuvieron un mejor rendimiento físico en salto horizontal, flexión de brazos en barra, carrera $5 \times 10$ $\mathrm{m}$, encogimiento abdominal y Course-Navette. Los sujetos con exceso de peso tuvieron un mejor rendimiento físico en dinamometría manual. No se observaron diferencias en los test de las placas y sit and reach.

En el trabajo de Castro-Piñero et al. ${ }^{23}$ se analizó en 1.513 varones y 1.265 mujeres de 6-17,9 años las diferencias en la fuerza muscular según el estatus de peso (bajo peso, normo-peso, sobrepeso y obesidad) clasificado a partir del $\mathrm{IMC}^{21}$; además, determinó valores percentiles de la fuerza muscular. La fuerza explosiva se midió con los test de lanzamiento de peso, salto horizontal y salto vertical. La fuerza resistencia se midió con los test de flexiones de brazos en el suelo, flexión de brazos en barra, dominadas, y tres test de encogimiento abdominal (máximas repeticiones, máximas repeticiones en 30 segundos y máximas repeticiones según un ritmo establecido). Los escolares y adolescentes con bajo peso y normo-peso mostraron niveles de fuerza similares. Los escolares y los adolescentes con bajo peso y normo-peso tuvieron un mejor desempeño físico en los test de fuerza explosiva de la parte inferior. Los escolares varones con bajo peso y normo-peso mostraron un mejor rendimiento físico en la prueba de flexiones de brazos en el suelo; y los escolares varones y las mujeres en el test de flexión de brazos en barra.

De la Cruz \& Pino ${ }^{24}$ examinaron en 137 varones y 156 mujeres españolas de 9,99 \pm 0,79 años, la relación entre diferentes dimensiones de la condición física, la obesidad y la actividad física en escolares. La condición física se midió con una versión abreviada de la batería Eurofit. Se establecieron dos grupos de escolares: baja condición física ( $\leq$ percentil 25$)$ y alta condición física ( $\geq$ percentil 75). El estatus de peso (normo-peso y exceso de peso) se categorizó a partir del $\mathrm{IMC}^{21}$. La actividad física se estimó con un registro de siete días ${ }^{25}$. Se clasificó a los escolares en dos grupos: suficientemente activos (cumplen las recomendaciones: $\geq 60$ minutos, $\geq 5$ días/semana de actividad física moderada o vigorosa, $\geq 3$ METS) e insuficientemente activos (no cumplen estas recomendaciones) ${ }^{26}$. Los escolares con normo-peso mostraron un mejor rendimiento físico en el test de Course-Navette, carrera $5 \times 10 \mathrm{~m}$ y salto horizontal. Los escolares con exceso de peso tuvieron un mejor desempeño en dinamometría manual. No se encontraron diferencias en el test de sit and reach. Además, aquellos con mejor condición física parecen cumplir, en mayor medida, las recomendaciones de actividad física.

La investigación de García-Artero et al. ${ }^{27}$ analizó en 1.196 varones y 1.278 mujeres las diferencias en la condición física según el estatus de peso (bajo peso, normo-peso, sobrepeso y obesidad) categorizado según el IMC ${ }^{21}$. Se midió 
la masa grasa y la magra. El bajo peso se asoció con un mejor rendimiento físico en el test de flexión de brazos en barra en las mujeres; y con un peor rendimiento físico en dinamometría manual en ambos sexos. Los sujetos con sobrepeso y obesidad mostraron un mejor rendimiento físico en el test de dinamometría manual; sin embargo, presentaron un peor rendimiento físico en los test de Course-Navette, carrera $4 \times 10 \mathrm{~m}$, salto horizontal y flexión de brazos en barra. En los test de Course-Navette, carrera $4 \times 10 \mathrm{~m}$, flexión de brazos en barra y salto horizontal no se encontraron diferencias estadísticamente significativas tras ajustar por la masa grasa.

En el estudio de Maestre et al. ${ }^{28}$ se analizó en 83 mujeres y 90 varones de diferentes razas (43 ingleses, 71 españoles, 29 sudamericanos y 30 niños de distintos países europeos, asiáticos y africanos) el nivel de condición física (mínimo, ideal y medio) según el estatus de peso (bajo peso, normopeso, sobrepeso y obesidad) categorizado a partir del IMC ${ }^{21}$. El nivel de condición física se estableció a partir del $\mathrm{VO}_{2}$ máx. que fue estimado mediante los resultados del test de Course-Navette. Los sujetos con normo-peso mostraron un mejor rendimiento en el test de Course-Navette así como valores superiores en $\mathrm{VO}_{2}$ máx. que sus semejantes con sobrepeso u obesidad. Los sujetos con sobrepeso mostraron un mejor rendimiento en el test de dinamometría manual. El metabolismo basal fue menor en el grupo de obesidad que en los grupos de bajo peso y normo-peso, y menor en el grupo de sobrepeso que en los grupos de bajo peso y normo-peso. Los sujetos son bajo peso y obesidad mostraron un nivel de condición física mínimo (tener en al menos dos variables valores en un tercil inferior).

En la investigación de Joshi et al. ${ }^{29}$ se determinó la relación entre obesidad y condición física. Se midió el IMC para categorizar a los sujetos con normopeso, sobrepeso y obesidad ${ }^{21}$. Se utilizaron los test del Fitnessgram ${ }^{\circledR 30}$ para medir distintos parámetros de la condición física (Course-Navette, alcanzar con las manos por la espalda, flexiones de brazos en el suelo y flexiones de tronco). Los sujetos con un IMC en normo-peso mostraron en general un mejor nivel de condición física.

El trabajo de Castro-Piñero et al. ${ }^{31}$ estudió en 1.491 varones y 1.261 mujeres de 6-17,9 años las diferencias en la capacidad aeróbica según el estatus de peso (bajo peso, normo-peso, sobrepeso y obesidad) clasificado a partir del IMC ${ }^{21}$; además, determinó valores percentiles de la condición cardiovascular. La capacidad aeróbica se midió con los test de Course-Navette, y las pruebas de correr/caminar una milla, una media milla y un cuarto de milla. Los sujetos con bajo peso mostraron un rendimiento similar a sus homólogos con peso normal. Los sujetos obesos o con sobrepeso tuvieron un menor rendimiento que sus homólogos con bajo peso o con peso normal.

El estudio de Mayorga-Vega et al. ${ }^{32}$ analizó en 36 varones y 35 mujeres la asociación entre el estatus del peso en base al índice de masa corporal (IMC) y la condición física. Se categorizó a los escolares en no sobrepeso y sobrepesoobesidad $^{21}$. Los sujetos con no sobrepeso obtuvieron valores más positivos en los test de Course-Navette, salto horizontal así como en peso, masa grasa, 
pliegues cutáneos y $\mathrm{VO}_{2}$ máx. No se observaron diferencias en el test de dinamometría manual.

El estudio de Arriscado et al. ${ }^{33}$ analizó en 161 varones y 168 mujeres de $11,7 \pm 0,4$ años, las relaciones de la condición física con la composición corporal, la tensión arterial y diversos factores sociodemográficos. Se categorizó a los sujetos según el IMC en normo-peso y sobrepeso/obesidad ${ }^{21}$. Se midieron los pliegues cutáneos, el perímetro de cintura y cadera (índice cintura/cadera) y se registraron valores de masa grasa. La condición física se midió con pruebas de la batería ALPHA-fitness ${ }^{34}$ incluyendo los estadios de maduración sexual de Tanner. Se añadió el test de flexión de tronco en posición de sentado. La presión arterial se midió con un esfigmomanómetro. Los varones y los sujetos con normo-peso obtuvieron rendimientos superiores en las pruebas de condición física que las mujeres y aquellos con sobrepeso u obesidad. Un mayor riesgo de padecer sobrepeso u obesidad se relacionó con un menor rendimiento en las pruebas de condición física, observándose relaciones inversas entre el porcentaje graso y el $\mathrm{VO}_{2}$ máx., y los test de salto horizontal y carrera 4 x $10 \mathrm{~m}$.

El trabajo de Rosa et al. ${ }^{35}$ estudió en 139 varones y 149 mujeres de 8-12 años, la relación entre el estatus de peso (normo-peso, sobrepeso y obesidad) determinado a partir del $I \mathrm{IC}^{21}$ y la capacidad aeróbica medida a partir del test de Course-Navette. Los sujetos con normo-peso mostraron significativamente un mejor rendimiento físico que sus semejantes con sobrepeso u obesidad

Gálvez et al. ${ }^{36}$ analizaron el nivel de condición física de 91 varones y 125 mujeres de 8-11 años en función de su estatus de peso categorizado a partir del IMC (normo-peso, sobrepeso y obesidad) ${ }^{21}$. Los parámetros de condición física se evaluaron mediante la batería ALPHA-fitness ${ }^{34}$ (carrera $4 \times 10 \mathrm{~m}$, CourseNavette, salto horizontal y dinamometría manual). Se calculó una medida global de la condición física. Los sujetos con un estatus de normo-peso mostraron un nivel más alto de condición física en comparación con sus pares homólogos con un estatus de sobrepeso u obesidad.

En otro trabajo de Rosa et al. ${ }^{37}$ se examinó en 114 varones y 142 mujeres la relación entre la composición corporal y la condición física. Se categorizó a los sujetos según el IMC con normo-peso, sobrepeso y obesidad. Se emplearon pruebas de la batería ALPHA-fitness para medir la condición física. Se calcularon un índice de fuerza muscular (IFM) a partir de los test de dinamometría manual y salto horizontal; y la condición física general (CF-ALPHA) a partir de test de dinamometría manual, salto horizontal, carrera $4 \times 10 \mathrm{~m}$ y Course-Navette. Se estimó el metabolismo basal ${ }^{38}$ y el $\mathrm{VO}_{2}$ máx. Los sujetos con normo-peso mostraron una mejor CF-ALPHA y un mejor IFM que sus semejantes con exceso de peso; además de un menor metabolismo basal. Los sujetos con sobrepeso mostraron un mejor desempeño en el test de dinamometría manual.

En el estudio de Pacheco-Herrera et al. ${ }^{39}$ se examinó en 3.129 varones y 4.139 mujeres la condición física muscular en relación con indicadores de adiposidad (IMC, perímetro der cintura, índice cintura/talla y masa grasa). Se 
calculó un índice de fuerza general a partir de los resultados en los test de dinamometría manual y salto horizontal siendo el cuartil cuarto el de mayor desempeño físico. Los sujetos dentro de este cuartil cuarto presentaron menores valores en los marcadores de adiposidad que su contraparte del Q1.

La investigación de Lema et al. ${ }^{40}$ analizó en 234 varones y 270 mujeres la relación entre la condición física y algunos indicadores antropométricos de adiposidad (IMC, masa adiposa y perímetro de cintura). Se midieron los siguientes indicadores de la condición física: capacidad aeróbica (test de Course-Navette), flexibilidad (test sit and reach), fuerza explosiva de miembros inferiores (test de salto horizontal) y fuerza resistencia abdominal (test de encogimiento abdominal). Se incluyeron también algunas variables sociodemográficas (sexo, grupo de edad, localización geográfica -rural o urbano, y carácter de la institución -público o privado-). Los sujetos con sobrepeso mostraron un menor rendimiento físico en los test de salto horizontal y CourseNavette. Aquellos que tenían una adiposidad central elevada se desempeñaron peor en los test sit and reach y Course-Navette.

El trabajo de Muros et al. ${ }^{41}$ describió y estudió en 255 varones y 260 mujeres las relaciones entre composición corporal, actividad física y condición física. Las variables antropométricas medidas fueron el IMC y los pliegues cutáneos del tríceps y subescapular a partir de los cuales se estimó la masa grasa ${ }^{42}$. La actividad física se estimó con una versión adaptada al castellano del Physical Activity Questionnaire for Older Children. Los parámetros de condición física medidos fueron los test de Course-Navette, dinamometría manual y salto horizontal. Los sujetos con sobrepeso y obesidad (especialmente estos últimos) tuvieron un peor desempeño físico en los test de salto horizontal y dinamometría manual. Mostraron a su vez valores inferiores de $\mathrm{VO}_{2}$ máx. y nivel de actividad física así como valores menos saludables en el IMC y en el porcentaje de masa grasa.

Tabla 1. Resumen de los estudios incluidos en la revisión.

\begin{tabular}{|c|c|c|c|}
\hline Autor y fecha & $\begin{array}{c}\text { Diseño y } \\
\text { participantes }\end{array}$ & $\begin{array}{c}\text { Componentes } \\
\text { analizados }\end{array}$ & Instrumentos \\
\hline Chen et al. (2006). & $\begin{array}{l}\text { Estudio } \\
\text { longitudinal, } \\
13.935 \text { sujetos } \\
\text { (1999); 24.586 } \\
\text { sujetos (2001), } \\
\text { de Taiwán, 6- } \\
18 \text { años. }\end{array}$ & $\begin{array}{l}\text { Morfológico, } \\
\text { cardiovascular, } \\
\text { músculo-esquelético. }\end{array}$ & $\begin{array}{l}\text { Test de campo } \\
\text { y pruebas de } \\
\text { laboratorio. }\end{array}$ \\
\hline Casajús et al. (2007). & $\begin{array}{l}\text { Estudio } \\
\text { transversal, } \\
1068 \text { sujetos } \\
\text { de España, 7- } \\
12 \text { años. }\end{array}$ & $\begin{array}{l}\text { Morfológico, capacidad } \\
\text { cardiovascular, } \\
\text { músculo-esquelético, } \\
\text { motor y amplitud de } \\
\text { movimiento. }\end{array}$ & Test de campo. \\
\hline
\end{tabular}




\begin{tabular}{|c|c|c|c|}
\hline Autor y fecha & $\begin{array}{l}\text { Diseño y } \\
\text { participantes }\end{array}$ & $\begin{array}{l}\text { Componentes } \\
\text { analizados }\end{array}$ & Instrumentos \\
\hline $\begin{array}{l}\text { Castro-Piñero et al. } \\
\text { (2009). }\end{array}$ & $\begin{array}{l}\text { Estudio } \\
\text { transversal, } \\
2778 \text { sujetos } \\
\text { de España, 6- } \\
17,9 \text { años. }\end{array}$ & $\begin{array}{l}\text { Morfológico y } \\
\text { músculo-esquelético. }\end{array}$ & $\begin{array}{l}\text { Test } \\
\text { campo. }\end{array}$ \\
\hline $\begin{array}{l}\text { De la Cruz \& Pino, } \\
(2010) .\end{array}$ & $\begin{array}{l}\text { Estudio } \\
\text { transversal, } \\
293 \text { sujetos } \\
\text { de España, } \\
9,99 \pm 0,79 \\
\text { años. }\end{array}$ & $\begin{array}{l}\text { Morfológico, músculo- } \\
\text { esquelético, } \\
\text { cardiovascular, motor, } \\
\text { amplitud de } \\
\text { movimiento, calidad } \\
\text { de la dieta y actividad } \\
\text { física }\end{array}$ & $\begin{array}{lr}\text { Test } & \text { de } \\
\text { campo } & y \\
\text { medidas de } & \\
\text { auto-reporte. }\end{array}$ \\
\hline $\begin{array}{l}\text { García-Artero et al. } \\
(2010) \text {. }\end{array}$ & $\begin{array}{l}2474 \text { sujetos } \\
\text { de España, } \\
13-18,5 \text { años. }\end{array}$ & $\begin{array}{l}\text { Morfológico, motor, } \\
\text { músculo-esquelético, } \\
\text { cardiovascular y } \\
\text { amplitud de } \\
\text { movimiento }\end{array}$ & $\begin{array}{l}\text { Test } \\
\text { campo. }\end{array}$ \\
\hline Maestre et al. (2010). & $\begin{array}{l}\text { Estudio } \\
\text { transversal, } \\
173 \text { sujetos } \\
\text { de España, 9- } \\
13 \text { años. }\end{array}$ & $\begin{array}{l}\text { Morfológico, músculo- } \\
\text { esquelético, cardio- } \\
\text { vascular, metabólico y } \\
\text { motor. }\end{array}$ & $\begin{array}{lr}\text { Test } & \text { de } \\
\text { campo } & y \\
\text { ecuaciones. }\end{array}$ \\
\hline $\begin{array}{l}\text { Castro-Piñero et al. } \\
\text { (2011). }\end{array}$ & $\begin{array}{l}\text { Estudio } \\
\text { transversal, } \\
2752 \text { sujetos } \\
\text { de España, 6- } \\
17,9 \text { años. }\end{array}$ & $\begin{array}{l}\text { Morfológico } \\
\text { capacidad } \\
\text { cardiovascular. }\end{array}$ & $\begin{array}{l}\text { Test } \\
\text { campo. }\end{array}$ \\
\hline Joshi et al. (2012). & $\begin{array}{l}\text { Estudio } \\
\text { transversal, } \\
7.000 \text { sujetos } \\
\text { de EE.UU. }\end{array}$ & $\begin{array}{l}\text { Morfológico, } \\
\text { cardiovascular } \\
\text { amplitud } \\
\text { movimiento. }\end{array}$ & $\begin{array}{l}\text { Test } \\
\text { campo. }\end{array}$ \\
\hline $\begin{array}{l}\text { Mayorga-Vega et al. } \\
\text { (2012). }\end{array}$ & $\begin{array}{l}\text { Estudio } \\
\text { transversal, } \\
71 \text { escolares } \\
\text { de España, } \\
\text { 10-12 años. }\end{array}$ & $\begin{array}{l}\text { Morfológico, músculo- } \\
\text { esquelético y } \\
\text { cardiovascular }\end{array}$ & $\begin{array}{l}\text { Test } \\
\text { campo. }\end{array}$ \\
\hline $\begin{array}{l}\text { Arriscado et al. } \\
\text { (2014). }\end{array}$ & $\begin{array}{l}329 \text { sujetos } \\
\text { de } \text { España, } \\
11-12 \text { años }\end{array}$ & $\begin{array}{l}\text { Morfológico, músculo- } \\
\text { esquelético, motor y } \\
\text { cardiovascular }\end{array}$ & $\begin{array}{l}\text { Test } \\
\text { campo. }\end{array}$ \\
\hline Rosa et al. (2014). & $\begin{array}{l}298 \text { sujetos } \\
\text { de España, 8- } \\
12 \text { años. }\end{array}$ & $\begin{array}{l}\text { Morfológico } \\
\text { cardiovascular }\end{array}$ & $\begin{array}{l}\text { Test } \\
\text { campo. }\end{array}$ \\
\hline
\end{tabular}




\begin{tabular}{|c|c|c|c|}
\hline Autor y fecha & $\begin{array}{c}\text { Diseño y } \\
\text { participantes }\end{array}$ & $\begin{array}{l}\text { Componentes } \\
\text { analizados }\end{array}$ & Instrumentos \\
\hline Gálvez et al. (2015). & $\begin{array}{l}216 \text { sujetos } \\
\text { de España, 8- } \\
11 \text { años }\end{array}$ & $\begin{array}{l}\text { Morfológico, } \\
\text { músculo- } \\
\text { esquelético, motor } \\
\text { y cardiovascular. }\end{array}$ & $\begin{array}{l}\text { Test } \quad \text { de } \\
\text { campo. }\end{array}$ \\
\hline Rosa et al. (2015). & $\begin{array}{l}256 \text { sujetos } \\
\text { de España, 8- } \\
11 \text { años. }\end{array}$ & $\begin{array}{l}\text { Morfológico, } \\
\text { músculo- } \\
\text { esquelético, motor, } \\
\text { cardiovascular y } \\
\text { metabólico }\end{array}$ & $\begin{array}{lr}\text { Test } & \text { de } \\
\text { campo } \quad y \\
\text { ecuaciones. }\end{array}$ \\
\hline $\begin{array}{l}\text { Pacheco-Herrera et al. } \\
\text { (2016). }\end{array}$ & $\begin{array}{l}\text { Estudio } \\
\text { transversal, } \\
7268 \text { sujetos } \\
\text { de Colombia, } \\
\text { 9-17,9 años. }\end{array}$ & $\begin{array}{l}\text { Morfológico } \\
\text { músculo- } \\
\text { esquelético. }\end{array}$ & $\begin{array}{l}\text { Test } \quad \text { de } \\
\text { campo y } \\
\text { bioimpedancia } \\
\text { eléctrica. }\end{array}$ \\
\hline Lema et al. (2016). & $\begin{array}{l}\text { Estudio } \\
\text { transversal, } \\
534 \text { sujetos } \\
\text { de Colombia, } \\
\text { 6-12 años }\end{array}$ & $\begin{array}{l}\text { Morfológico, } \\
\text { cardiovascular, } \\
\text { músculo- } \\
\text { esquelético } \\
\text { amplitud } \\
\text { movimiento }\end{array}$ & $\begin{array}{l}\text { Test } \\
\text { campo }\end{array}$ \\
\hline Muros et al. (2016). & $\begin{array}{l}\text { Estudio } \\
\text { transversal, } \\
515 \text { sujetos } \\
\text { de Colombia, } \\
10,6 \pm 0,5 \\
\text { años. }\end{array}$ & $\begin{array}{l}\text { Morfológico, } \\
\text { músculo- } \\
\text { esquelético } \\
\text { cardiovascular. } \\
\text { Actividad física }\end{array}$ & $\begin{array}{l}\text { Test de } \\
\text { campo, } \\
\text { ecuaciones y } \\
\text { medidas de } \\
\text { auto-reporte }\end{array}$ \\
\hline
\end{tabular}

Fuente: elaboración propia.

\section{DISCUSIÓN}

En la discusión de los resultados de estos estudios es necesario tener en cuenta tres aspectos: 1) Se han empleado diseños de tipo observacional, longitudinal y experimental; 2) Se han analizado muestras de entornos socioculturales procedentes de América del Norte, Sudamérica, Europa y Asia; 3) Se han empleado distintas baterías de test de campo y, en menor medida, pruebas de laboratorio. Al analizar los resultados de estos estudios se desprende que los sujetos con un IMC elevado tienen un menor rendimiento físico en la mayoría de los test de valoración de la salud relacionada con la CF. Al categorizar a los sujetos según su $\mathrm{IMC}^{21}$, aquellos con un estatus no saludable (sobre todo, con obesidad) manifiestan una mayor tendencia a encontrarse en percentiles inferiores en diversos componentes de la CF, especialmente en condición cardiovascular. 


\section{Estatus de peso y condición física general}

En algunos estudios ${ }^{36-37}$ se calculó una medida global de la CF a partir de los resultados registrados en los test que medían los indicadores de velocidadagilidad, fuerza de prensión manual, fuerza explosiva del tren inferior y capacidad aeróbica (CF-ALPHA, 0-10). Los resultados mostraron que aquellos sujetos con una mayor tendencia a poseer un estatus de sobrepeso u obesidad presentaban un bajo nivel de condición física (CF-ALPHA =3,9 $\pm 1,7$ ).

Los resultados reportados en otro de los trabajos indican que los sujetos con bajo peso también muestran una tendencia a asociarse con una condición física muy mala (< tercil inferior en al menos dos pruebas) concluyendo que los extremos (bajo peso y obesidad) son igual de peligrosos ${ }^{28}$. En otros trabajos ${ }^{23,31}$ los sujetos con bajo peso y normo-peso tuvieron valores similares en las pruebas que medían la capacidad aeróbica y la fuerza muscular. Por tanto, en lo que respecta al rendimiento físico en pruebas como la Course-Navette, el salto horizontal y las flexiones de brazos en el suelo, tener un estatus de bajo peso podría no ser un factor limitante de la capacidad física del sujeto siendo necesario analizar otros factores determinantes como la cantidad de masa muscular. Otro parámetro a tener en cuenta podría ser la realización de actividad física. Un estudio ${ }^{24}$ reportó que los sujetos que cumplían las recomendaciones de actividad física ${ }^{26}$ tenían un estatus de no sobrepeso y un nivel alto de condición física ( $\geq$ percentil 75 en cada variable) frente a aquellos que no las cumplían y mostraban una mayor tendencia a un estatus con exceso peso y una baja condición física ( $\leq$ percentil 25 en cada variable).

\section{Estatus de peso y capacidad aeróbica}

En comparación con sus semejantes con un estatus de sobrepeso u obesidad, los sujetos con bajo peso ${ }^{31}$ o con normo-peso $20,22,24,27-29,32-33,35-37$ tenían niveles significativamente superiores de capacidad aeróbica medida en metros recorridos 29,31 minutos $^{31}$, o paliers ${ }^{22,24,32-33,35-36,40}$ que sus pares homólogos con sobrepeso u obesidad.

Aunque un IMC indicativo de exceso de peso no implica necesariamente una habilidad reducida para consumir oxígeno de forma máxima ${ }^{44}$, se ha descrito que la capacidad aeróbica suele encontrarse inversamente relacionada con la cantidad total de masa corporal, probablemente como consecuencia del incremento de la carga inerte producida por el exceso de grasa ${ }^{45}$. Algunos estudios demuestran que este hecho sucede de manera evidente en la infancia y la adolescencia ya que los sujetos con sobrepeso u obesidad tienen valores inferiores de $\mathrm{VO}_{2}$ máx. ${ }^{32-33,41}$ con respecto a sus semejantes con normo-peso. En este sentido, se ha comentado anteriormente descrito que sujetos con valores muy reducidos de $\mathrm{VO}_{2}$ máx. tienen una mayor tendencia a poseer una condición física baja o muy mala (< tercil inferior de, al menos, dos pruebas) ${ }^{28}$.

No obstante, en el estudio de Chen et al. ${ }^{20}$ se observó que el riesgo de hipertensión era significativamente menor si los sujetos con sobrepeso/obesidad 
tenían un nivel de aptitud cardiovascular más alto en comparación con sus semejantes con un nivel inferior al promedio. Además, se ha descrito que sujetos con un IMC indicativo de sobrepeso u obesidad podían presentar niveles de condición física aceptables si tenían valores de $\mathrm{VO}_{2}$ máx. saludables. Es lo que se ha descrito como fat but fit $u$ obeso, pero en forma ${ }^{46}$. Los resultados del estudio sugieren que es el nivel de capacidad aeróbica y no tanto el grado de actividad física, el parámetro que determina en mayor medida la salud cardiovascular, así como predice factores de riesgo cardiovascular futuro. No obstante, los resultados aportados en algunos de los trabajos de esta revisión ${ }^{20,24}$ confirman que la realización de actividad física se muestra como la estrategia más eficaz para tener una CF y un estatus saludable.

\section{Estatus de peso y fuerza muscular}

En el estudio de Pacheco-Herrera et al. ${ }^{39}$ se observó que los sujetos con un índice de fuerza general superior (evaluado con los test de dinamometría manual y salto horizontal) tenían valores antropométricos más saludables (IMC, perímetro de cintura, índice cintura/talla y masa grasa) que sus semejantes con un índice de fuerza general inferior. Esto coincide con lo reportado en el trabajo de Rosa et al. en el cual se emplearon similares instrumentos de medición.

En el análisis de los indicadores de la fuerza muscular, diversas investigaciones muestran de manera inequívoca que los sujetos con normo-peso tienen una mayor potencia explosiva en el tren inferior independientemente del test de valoración empleado ${ }^{23}$. Es en el estudio de la fuerza del tren superior donde se encuentran resultados contradictorios. En las pruebas de fuerza prensil los estudios indican que los sujetos con sobrepeso se muestran más competentes que sus semejantes con normo-peso y obesidad 24,28,33,36,37.

Aunque los sujetos con sobrepeso puedan presentar porcentajes superiores de grasa corporal también pueden tener mayores porcentajes de masa muscular, lo que no impide e incluso favorece su rendimiento en este tipo de pruebas. Si se emplean pruebas en las que la inercia juega un papel muy relevante en el rendimiento físico del individuo como el test de lanzamiento de peso, los resultados encontrados son similares ${ }^{23}$. Por el contrario, en las pruebas en las que es necesario propulsar la masa corporal como el test de flexión de brazos los sujetos con sobrepeso y obesidad se muestran menos competentes que sus semejantes con bajo peso y sobre todo con respecto a los sujetos con normo-peso $22-23,27$.

\section{Estatus de peso y capacidad motora}

Los resultados de diversos trabajos confirman que un IMC de sobrepeso/obesidad se relaciona de manera inversa con la velocidad-

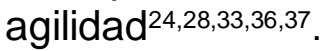

Tal y como sucede en los test de valoración de la capacidad aeróbica y en algunos test de fuerza muscular, los sujetos con mayor masa corporal presentan una evidente desventaja ya que deben mover una carga extra durante la ejecución de estas pruebas. 


\section{Estatus de peso y flexibilidad}

Aunque la sabiduría coloquial sugiere que un IMC elevado se asocia con una menor amplitud de movimiento, algunos trabajos no han reportado diferencias significativas en la flexibilidad entre sujetos con normo-peso y aquellos con sobrepeso/obesidad $22,24,29$. Sin embargo, en un estudio se reportó que aquellos que tenían una adiposidad central elevada se desempeñaron peor en el test sit and reach.

\section{Estatus de peso y metabolismo basal}

En algunos trabajos ${ }^{28,35}$ se observó que el metabolismo basal fue menor en los grupos de sobrepeso y obesidad que en los grupos de bajo peso y normopeso, necesitando estos sujetos una mayor cantidad de energía para que su cuerpo subsista.

\section{CONCLUSIONES}

Las conclusiones de esta revisión son las siguientes: 1) Un estatus de obesidad se asocia a un nivel no saludable de CF; 2) Un estatus de bajo peso podría ser tan peligroso como un estatus de obesidad; 3) Un estatus de normopeso se asocia a un mejor nivel de capacidad aeróbica, potencia explosiva del tren inferior, fuerza resistencia abdominal, fuerza resistencia del tren superior, y velocidad-agilidad, así como a valores más positivos de $\mathrm{VO}_{2}$ máx. y metabolismo basal en comparación con el sobrepeso y la obesidad; 4) Un estatus de sobrepeso se asocia a un mejor nivel de fuerza prensil con respecto al bajo peso, normo-peso y obesidad.

\section{APLICACIONES PRÁCTICAS}

En base a los resultados de esta revisión se confirma que es necesario considerar las siguientes pautas en el diseño de programas de intervención: 1) El objetivo primordial debe ser educar a los sujetos para la actividad física y para la mejora de la condición física; 2) Las intervenciones deben favorecer el desarrollo de, al menos, dos indicadores de la condición física siendo uno de ellos la capacidad aeróbica; 3) Un objetivo importante debe ser alcanzar un estatus de normo-peso. En los casos en los que no sea posible, el objetivo será alcanzar percentiles elevados de capacidad aeróbica; 4) El recurso principal en todas las intervenciones debe ser la realización de actividad física. Las recomendaciones actuales ( $\geq 60$ minutos, $\geq 5$ días/semana de actividad física moderada o vigorosa, $\geq 3$ METS) son efectivas; 5 ) Teniendo en cuenta que con la legislación actual solo se realizan dos sesiones semanales de educación física y ante la imposibilidad de algunos sujetos de cumplir con las recomendaciones anteriores, la escuela parece ser el marco ideal para potenciales iniciativas en este sentido. Los profesores de educación física están formados para valorar al alumnado y desarrollar programas de mejora de la condición física. Con la finalidad de reducir costes sanitarios y mejorar la salud pública, es fundamental 
incrementar la carga lectiva semanal. Asimismo, sería importante incentivar adecuadamente al profesorado para llevar a la práctica programas de deporte escolar así como investigaciones que contribuyan al conocimiento científico.

\section{REFERENCIAS}

1. LaMonte MJ, Barlow CE, Jurca R, Kampert JB, Church TS, Blair SN. Cardiorespiratory fitness is inversely associated with the incidence of metabolic syndrome: a prospective study of men and women. Circulation. 2005; 112: 505-12.

2. Carnethon MR, Gidding SS, Nehgme R, Sidney S, Jacobs DR Jr, Liu K. Cardiorespiratory fitness in young adulthood and the development of cardiovascular disease risk factors. JAMA. 2003; 290: 3092-100.

3. Balady GJ. Survival of the fittest-more evidence. N Engl J Med. 2002; 346 (11): 852-4. 5.

4. Castillo MJ. Physical fitness is an important contributor to health for the adults of tomorrow. Selección. 2007; 17 (1): 2-8.

5. Gulati M, Pandey DK, Arnsdorf MF, Lauderdale DS, Thisted RA, Wicklund RH, et al. Exercise capacity and the risk of death in women: the St James Women Take Heart Project. Circulation. 2003; 108 (13): 1554-9.

6. Laukkanen JA, Lakka TA, Rauramaa R, Kuhanen R, Venalainen JM, Salonen R, et al. Cardiovascular fitness as a predictor of mortality in men. Arch Intern Med. 2001; 161(6): 82531.

7. Rosa-Guillamón A. \& García-Cantó E. (2016). Relationship between physical fitness and mental health in primary school children. Revista Iberoamericana de Ciencias de la Actividad Física y el Deporte. 2016; 5(2): 31-42.

8. Rosa-Guillamón A, García-Cantó E, Rodríguez-García PL, Pérez-Soto JJ. Weight status, physical fitness and satisfaction with life among elementary school children. a pilot study. (2016). Revista MHSalud. 2017; 13(2): 1-15.

9. Ruiz JR, Castro-Pinero J, Artero EG, Ortega FB, Sjostrom M, Suni J, Castillo MJ. Predictive validity of health-related fitness in youth: a systematic review. $\mathrm{Br}$ J Sports Med. 2009; 43(12): 909-923.

10. Kim J, Must A, Fitzmaurice GM, Gillman MW, Chomitz V, Kramer E, McGowan R, Peterson KE. Relationship of physical fitness to prevalence and incidence of overweight among schoolchildren. Obes Res. 2005; 13(7): 1246-54.

11. Mota J, Ribeiro JC, Carvalho J, Santos MP, Martins J. Cardiorespiratory fitness status and body mass index change over time: a 2-year longitudinal study in elementary school children. Int J Pediatr Obes. 2009; 4(4): 338-42.

12. Coelho-E-Silva MJ, Vaz Ronque ER, Cyrino ES, Fernandes RA, Valente-Dos-Santos J, Machado-Rodrigues A, Martins R, Figueiredo AJ, Santos R, Malina RM. Nutritional status, biological maturation and cardiorespiratory fitness in Azorean youth aged 11-15 years. BMC Public Health. 2013; 13: 495.

13. Rush E, McLennan S, Obolonkin V, Vandal, AC, Hamlin M, Simmons D, Graham D. Project Energize: whole-region primary school nutrition and physical activity programme; evaluation of body size and fitness 5 years after the randomised controlled trial. $\mathrm{Br} \mathrm{J}$ Nutr. 2014; 111 (2): 363-371.

14. Mata J, Silva MN, Vieira PN, Carraça EV, Andrade AM, Coutinho SR, Teixeira PJ. Motivational "spill-over" during weight control: Increased self-determination and exercise intrinsic motivation predict eating self-regulation. Sport, Exercise, and Performance Psychology. 2011; 1(Suppl), 49-59.

15. Kozub FM. Motivation and Physical Activity in Adolescents With Visual Impairments: Review: Rehabilitation and Education for Blindness and Visual Impairment. 2006; 37 (4): 149160.

16. Teixeira PJ, Going SB, Houtkooper LB, Cussler EC, Metcalfe LL, Blew RM \& Lohman TG. Exercise motivation, eating, and body image variables as predictors of weight control. Medicine and Science in Sports and Exercise. 2006 38(1): 179-188. 
17. Han JC, Lawlor DA, Kimm SY. Childhood obesity. The Lancet. 2010; 375 (9727): 17371748.

18. Klein D, De Toia D, Weber S, Wessely N, Koch B, Dordel S, Graf, C. Effects of a low threshold health promotion intervention on the BMI in pre-school children under consideration of parental participation. E-SPEN, the European e-Journal of Clinical Nutrition and Metabolism. 2010; 5 (3): e125-e131.

19. Rodríguez-Hernández A, Feu S, Martínez-Santos R, de la Cruz-Sánchez E. ebalonmano.com: Revista de Ciencias del Deporte. 2011; 7 (3): 157-168.

20. Chen LJ, Fox KR, Haase A, Wang JM. Obesity, fitness and health in Taiwanese children and adolescents. European Journal of Clinical Nutrition. 2006; 60: 1367-1375.

21. Cole TJ, Bellizzi MC, Flegal KM, Dietz WH. Establishing a standard definition for child overweight and obesity worldwide: international survey. British Medical Journal. 2000; 320: 1240-1243.

22. Casajús JA, Leiva MT, Villarroya A, Legaz A, Moreno LA. Physical performance and school physical education in overweight Spanish children. Ann Nutr Metab. 2007;51(3):28896.

23. Castro-Piñero J, González-Montesinos JL, Mora J, Keating XD, Ruiz J. Percentile values for muscular strength field tests in children aged 6 to 17 years: influence of weight status. Journal of Strength Cond Res. 2009; 23(8): 2295-310.

24. De la Cruz E, Pino J. (2010). Health-related physical fitness in schoolchildren and Spanish Physical Activity Guidelines. Cultura, Ciencia y Deporte. 2010; 5: 45-49.

25. Bratteby LE, Sandhagen B, Fan H, Samuelson G. A 7-day activity diary for assessment of daily energy expenditure validated by the doubly labelled water method in adolescents. European Journal of Clinical Nutrition. 1999; 51(9): 585-591.

26. Department of Health, Physical Activity, Health Improvement and Prevention. At least five a week: evidence on the impact of physical activity and its relationship to health. A report from the Chief Medical Officer. London: Department of Health; 2004.

27. García-Artero EG, España-Romero V, Ortega FB, Jiménez-Pavón D, Ruiz JR, VicenteRodríguez G, Bueno M, Marcos A, Gómez-Martínez S, Urzanqui A, González-Gross M, Moreno LA, Gutiérrez A, Castillo MJ. Health-related fitness in adolescents: underweight, and not only overweight, as an influencing factor. The AVENA study. Scandinavian Journal of Medicine \& Science in Sports. 2010; 20: 418-427.

28. Maestre JM. Connection between nutritional state and physical fitness in schoolar population. Journal of Sport and Health Research. 2010; 2(2), 95-108.

29. Joshi $P$, Bryan $C$, Howat $H$. Relationship of body mass index and fitness levels among schoolchildren. J Strength Cond Res. 2012; 26 (4): 1006-14.

30. Cooper Institute for Aerobics Research. FITNESSGRAM Test Administrator's Manual, 2nd. Ed. Champaign, II: Human Kinetics; 1999.

31. Castro-Piñero J, Mora J, González-Montesinos JS, Ortega FB, Keating XD, Sjöström M, Ruiz RJ. Percentile values for cardiorespiratory fitness running/walking field tests in children aged 6 to 17 years. Nutr Hosp. 2011; 26(3): 572-578.

32. Mayorga-Vega D, Brenes-Podadera A, Rodríguez-Tejero M, Merino-Marban R. Association of BMl and physical fitness level among elementary school students. Journal of Sport and Health Research. 2012; 4(3): 299-310.

33. Arriscado D, Dalmau JM, Muros JJ, Zabala M. Relación entre condición física y composición corporal en escolares de primaria del norte de España. Nutr Hosp. 2014; 30(2): 385-394.

34. Ruiz JR, España Romero V, Castro Piñero J, Artero EG, Ortega FB, Cuenca García, M, et al. Batería ALPHA-Fitness: test de campo para la evaluación de la condición física relacionada con la salud en niños y adolescentes. Nutr Hosp. 2011; 26 (6): 1210-1214.

35. Rosa A, García-Cantó E, Rodríguez-García PL, Pérez-Soto JJ. Nivel de capacidad aeróbica y su relación con el estatus corporal en escolares de 8 a 12 años. EmásF, Revista Digital de Educación Física. 2014; 6(31): 7-20.

36. Gálvez A, Rodríguez-García PL, Rosa A, García-Cantó E, Pérez-Soto JJ, Tárraga ML, Tárraga PJ. Nivel de condición física y su relación con el estatus de peso corporal en escolares. Nutr Hosp. 2015; 31(1): 393-400. 
37. Rosa A, Rodríguez-García PL, García-Cantó E, Pérez-Soto JJ. Niveles de condición física de escolares de 8 a 11 años en relación al género y a su estatus corporal. Ágora para la EF y el Deporte. 2015; 17(3): 237-250.

38. Neri M, Bargossi A. Alimentación y ciclismo. Bilbao: Dorleta. 2000.

39. Pacheco-Herrera JD, Ramírez-Vélez R, Correa-Bautista JE. Índice general de fuerza y adiposidad como medida de la condición física relacionada con la salud en niños y adolescentes de Bogotá, Colombia: Estudio FUPRECOL. Nutr Hosp. 2016;33: 556-564

40. Lema L, Mantilla SC, Arango CM. Associations Between Physical Fitness and Adiposity Among School-Age Children from Monteria, Colombia. Rev int med cienc act fís deporte. 2016; 16(62): 277-296.

41. Muros JJ, Cofre-Bolados C, Salvador-Pérez, S, Castro-Sánchez M, Valdivia-Moral P, Pérez-Cortés A.J. (2016). Relación entre nivel de actividad física y composición corporal en escolares de Santiago (Chile). Journal of Sport and Health Research. 2016; 8(1):65-74.

42. Slaughter, M.H., Lohman, T.G., Boileau, R.A., Stillman, P.J., Van Loan, M.D., Bembem, D.A. (1988). Skinfolds equations for estimation of body fatness in children and youth. Human Biology. 60(5), 709-723.

43. Martínez-Gómez D, Martínez-De-Haro V, Pozo T, Welk GJ, Villagra A, Calle ME, Valcarce-Sánchez E. Reliability and validity of the PAQ-A questionnaire to assess physical activity in Spanish adolescents. Revista Española de Salud Pública. 2009; 83(3): 427-439.

44. Goran M, Fields DA, Hunter GR, Herd SL, Weinsier RL. Total body fat does not influence maximal aerobic capacity. Int J Obes Relat Metab Disord. 2000; 24: 841-8.

45. Mota J, Guerra S, Leandro C, Pinto A, Ribeiro JC, Duarte JA. Association of maturation, sex, and body fat in cardiorespiratory fitness. Am J Hum Biol. 2002; 14:707-12.

46. McAuley PA, Blair SN. Obesity paradoxes. Journal of Sports Sciences. 2011; 29(8); 773782.

Referencias totales citadas: 46

Referencias citadas correspondientes a la Rev Ib CC Act Fis Dep: 1 